Research Paper

\title{
Comparison between CT and MRI in the Diagnostic Accuracy of Thymic Masses
}

\author{
Hao-Ran Li1 ${ }^{1}$, Jian Gao' ${ }^{1}$, Chun Jin², Jia-Hao Jiang ${ }^{1}$, Jian-Yong Ding1, ${ }^{1,}$ \\ 1. Department of Thoracic Surgery, Zhongshan Hospital, Fudan University, 200032, Shanghai, China. \\ 2. Department of Thoracic Surgery, Xuhui District Center Hospital of Shanghai, 200031, Shanghai, China. \\ $\triangle$ Corresponding author: Jian-Yong Ding, M.D., Ph.D., Department of Thoracic Surgery, Zhongshan Hospital, Fudan University, 180 Fenglin Road, Xuhui \\ District, Shanghai 200032, P.R. China, Tel. \& Fax: +86-21-64041990, E-mail: dingjianyongmd@163.com \\ (C) Ivyspring International Publisher. This is an open access article distributed under the terms of the Creative Commons Attribution (CC BY-NC) license \\ (https://creativecommons.org/licenses/by-nc/4.0/). See http://ivyspring.com/terms for full terms and conditions.
}

Received: 2018.09.27; Accepted: 2019.04.15; Published: 2019.06.02

\begin{abstract}
Purpose: The aim of this study was to compare diagnostic accuracy between CT and MRI for thymic masses.

Methods: We searched literature and collected information on first author, publication year, cases of different types of thymic lesions, correct diagnostic cases of CT and MRI and results of quantitative analysis of CT and MRI. The ROC curve was applied to compare the diagnostic performance of different imaging modalities.

Results: Eight literatures were finally included and analyzed in this study. There were 253 cases examined by CT and 340 cases by MRI in total. We showed outcomes of quantitative analysis of each study in this article. The sensitivity of CT and MRI was both $100 \%$, while the specificity was $75 \%$ and $80 \%$, respectively. AUC of CT was 0.875 [95\% Cl: $0.473,0.997]$ and that of MRI was 0.880 [95\%Cl: 0.531, 0.995].

Conclusion: The diagnostic accuracy of MRI is superior to CT in detecting thymomas, thymic cysts or thymic hyperplasia but that of CT and MRI is still unclear in differentiating thymic carcinomas and lymphomas/germ cell tumors.
\end{abstract}

Key words: thymic mass, thymomas, CT, MRI, diagnostic accuracy

\section{Introduction}

Thymic masses are comprised of hyperplasia, cysts, thymic epithelial tumors (TETs), lymphomas, malignant germ cell tumors or metastatic cancers and so on $[1,2]$. True thymic hyperplasia is usually regarded as a rebound phenomenon and characterized by an increase in mass of the gland after a stressor, such as chemotherapy, radiation, steroid treatment, burns or surgery [3]. Thymic cysts are relatively uncommon lesions that can be found at any age and can be congenital or acquired [2,3]. Although TETs are rare neoplasms as to the whole tumors, they are the most common mediastinal tumors in adults, including thymomas and thymic carcinomas [4, 5]. The overall incidence of thymoma is 1.5 cases per million (www.nccn.org/). Thymoma is a slow-growing neoplasm that can invade to adjacent structures, including the pericardium and pleura, whereas distant metastases are rare [6]. Thymic carcinomas are far rarer but more aggressive than thymomas, thus thymic carcinomas is often found having invaded to adjacent structures and metastasized to distant organ [7].

Thymectomy is the main therapeutic method of thymic masses, while radiotherapy and chemotherapy are often as (neo)adjuvant and palliative procedures $[8,9]$. Generally, surgery is often performed under patients with paraneoplastic syndromes like myasthenia gravis $[6,10]$ or with thymic malignancy except for lymphoma. Therefore, patients would be overtreated or be in lack of essential therapy if thoracic surgeons could not differentiate the properties of thymic lesions. Imaging evaluation 
has been employed to initially diagnose and properly staging thymoma, especially, on the detection of direct invasion and distant organ metastasis [6].

Computed tomography (CT) is currently the first choice to identify and characterize thymic masses due to the high spatial, temporal resolution and convenience [11-13]. Moreover, CT does offer a higher sensitivity and specificity for detecting of thymic lesions regardless of high radioactivity [12]. Some CT imaging parameters were significantly correlated with stage, classification and the completeness of resection of thymomas, but none can reliably predict overall survival or disease-free survival duration [11]. Magnetic Resonance imaging (MRI) is not routinely used to detect mediastinal masses like TETs except for patients with iodine allergy or with renal failure [14, 15]. However, MRI is better in detecting cystic lesion like thymic cysts or distinguishing cystic from solid masses than CT [15]. The typical appearance of thymoma in MRI is low to intermediate signal intensity on T1-weighted sequences and high signal intensity on T2-weighted sequences [15]. MRI has different functional modes like Chemical shift MRI and diffusion-weighted magnetic resonance imaging, thereby it can show better image contrast [16]. Additionally, MRI does have an excellent contrast resolution [15] and can be a better tool when patients are suspected to have invasive thymoma with adjacent structures invasion $[17,18]$.

However, it remains unclear which diagnostic modality is the most accurate diagnostic tool for thymic lesions. Herein, we try to unveil which imaging tool is better in accuracy of diagnosis of thymic masses.

\section{Material and methods}

\section{Search strategy}

A literature search was conducted (through $1^{\text {st }}$, January, 2009 to $1^{\text {st }}$, April, 2018) to identify all articles that have been published about accuracy of pre-operative CT and MRI examination. The terms "computed tomography", "CT", "magnetic resonance imaging", "MRI", "diagnosis", "accuracy", "detection", "thymic masses", "thymomas", "thymic cysts", "anterior mediastinal mass" and "thymic carcinomas" were searched in the title, abstract or key words on PubMed/MEDLINE, Web of science and Cochrane Library.

\section{Inclusion and exclusion criteria}

The inclusion criteria were as follows: 1) cohort studies; 2) diagnostic comparison between MRI and CT (these two tools combined or single modality); 3 ) staging and classification of thymomas were according to World Health Organization Histologic
Classification [19] or Masaoka-Koga stage system [20]; 4) all thymic masses were confirmed by histopathological examination. Case reports, reviews, editorials, abstracts, comments were all excluded.

\section{Information collection}

We collected information from each study on first author, publication year, cases of different types of thymic lesions and results of quantitative analysis of CT and MRI. Additionally, we found out correct and erroneous cases of each imaging in detecting thymic lesions.

\section{Statistical analysis}

Statistical analyses were performed with SPSS 23.0 for Windows software. ROC (receiver operating characteristics) analysis was applied to compare the diagnostic capability of different imaging modalities of thymic masses. The differences between groups were analyzed using a Student's t-test. $P$ value $<0.05$ was considered as significant.

\section{Results}

\section{Publication screening}

Sixteen studies were obtained after applying search strategy and screening abstracts, and eight studies were included further applying inclusion criteria[2, 21-27] (Table 1). There were 253 cases on CT examination and 340 cases on MRI in total. On CT, these included 99 thymomas, 12 thymic carcinomas, 22 thymic cysts, 75 thymic hyperplasia, 25 lymphomas or germ cell tumors and 20 other masses like seminoma. On MRI, these included 139 thymomas, 15 thymic carcinomas, 12 thymic cysts, 95 thymic hyperplasia, 47 lymphomas or germ cell tumors and 32 other masses.

Table 1. Basic information of included studies

\begin{tabular}{llllll}
\hline Nation & Authors & Year & Cases & Mean age & Imaging \\
\cline { 5 - 6 } & & & & & modality \\
\hline United & B. Ackman et al. & 2014 & 160 & $51(15-87)$ & CT \\
States & T. Araki et al. & 2014 & 18 & $56(37-79)$ & CT \\
Korea & Chang et al. & 2017 & 37 & 54 & CT \\
Italy & Priola et al. & 2016 & 83 & $38.4(14-71)$ & CT/MRI \\
Japan & Seki et al. & 2014 & 35 & $51.6(22-75)$ & CT/MRI \\
Japan & Tomiyama et al. & 2009 & 127 & $45.9(10-82)$ & CT/MRI \\
Japan & Usuda et al. & 2015 & 16 & $*$ & MRI \\
Japan & H. Yabuuchi et al. & 2015 & 48 & $50.7(21-83)$ & MRI \\
\hline
\end{tabular}

*. unmentioned

\section{Quantitative assessment of CT and MRI}

Quantitative analysis was performed to compare percentage of correct diagnosis of thymic masses between CT and MRI. Percentage of correct diagnosis on CT is $77.78 \%$ in thymoma, $37.5 \%$ in thymic carcinoma, $61.36 \%$ in thymic cysts, $84.00 \%$ in thymic 
hyperplasia, $66.00 \%$ in lymphoma/germ cell tumor, and $57.50 \%$ in other mass (Table 2). While percentage of correct diagnosis on MRI is $88.13 \%$ in thymoma, $30.00 \%$ in thymic carcinoma, $70.83 \%$ in thymic cysts, $97.89 \%$ in thymic hyperplasia, $48.94 \%$ in lymphoma/germ cell tumor and $54.69 \%$ in other mass (Table 3).

Table 2. Percentage of correct diagnosis by CT for all thymic mass.

\begin{tabular}{lllll}
\hline & cases & correct & incorrect & No.(\%)correct \\
\hline Thymoma & 99 & 77 & 22 & $77.78 \%$ \\
Thymic carcinoma & 12 & 4.5 & 7.5 & $37.50 \%$ \\
Thymic cyst & 22 & 13.5 & 8.5 & $61.36 \%$ \\
Thymic hyperplasia & 75 & 63 & 12 & $84.00 \%$ \\
Lymphoma/germ cell tumor & 25 & 16.5 & 18.5 & $66.00 \%$ \\
Other mass & 20 & 11.5 & 8.5 & $57.50 \%$
\end{tabular}

Table 3. Percentage of correct diagnosis by MRI for all thymic mass.

\begin{tabular}{lllll}
\hline & cases & correct & incorrect & No.(\%)correct \\
\hline Thymoma & 139 & 122.5 & 16.5 & $88.13 \%$ \\
Thymic carcinoma & 15 & 4.5 & 10.5 & $30.00 \%$ \\
Thymic cyst & 12 & 8.5 & 3.5 & $70.83 \%$ \\
Thymic hyperplasia & 95 & 93 & 2 & $97.89 \%$ \\
Lymphoma/germ cell tumor & 47 & 23 & 24 & $48.94 \%$ \\
Other mass & 32 & 17.5 & 14.5 & $54.69 \%$ \\
\hline
\end{tabular}

Thymic cysts were generally 38 HU (Hounsfield unit, range 6-62 HU) on contrast-enhanced $\mathrm{CT}$ and are 45 HU (range 26-64 HU) on unenhanced CT. Table 4 showed quantitative assessment of CT in thymoma and thymic carcinoma by using $\mathrm{HU}$ and contrast $\mathrm{HU}$.

Table 4. Quantitative assessment of CT in thymoma and thymic carcinoma*.

\begin{tabular}{lll}
\hline Thymoma & mean Hounsfield unit & contrast Hounsfield unit \\
\hline low-risk thymoma & 29.78 & $65.8(59.55-86.83)$ \\
high-risk thymoma & 14.55 & $49.35(48.85-60.45)$ \\
thymic carcinoma & 19.95 & $57.45(55.75-65.50)$ \\
\hline *Data from Chang et 2017 &
\end{tabular}

Table 5. Quantitative assessment of MRI in different thymic masses.

\begin{tabular}{|c|c|c|c|c|c|}
\hline & $\begin{array}{l}\text { Yabuuchi } \\
\text { et al. } 2015\end{array}$ & $\begin{array}{l}\text { Usuda et } \\
\text { al. } 2015\end{array}$ & $\begin{array}{l}\text { Abdel Razek } \\
\text { et al. } 2014\end{array}$ & $\begin{array}{l}\text { Priola et al. } \\
2015\end{array}$ & $\begin{array}{l}\text { Priola et al. } \\
2015\end{array}$ \\
\hline Thymic mass & \multicolumn{4}{|c|}{$\mathrm{ADC}\left(\times 10-3 \mathrm{~mm}^{2} / \mathrm{s}\right)$} & SII (\%) \\
\hline $\begin{array}{l}\text { Low-risk } \\
\text { thymoma }\end{array}$ & $1.03 \pm 0.38$ & $1.71 \pm 0.48$ & $1.30 \pm 0.08$ & $1.53 \pm 0.25$ & $-0.81 \pm 3.56$ \\
\hline $\begin{array}{l}\text { High-risk } \\
\text { thymoma }\end{array}$ & $1.04 \pm 0.27$ & & $1.16 \pm 0.07$ & $1.09 \pm 0.24$ & $1.18 \pm 4.20$ \\
\hline Thymic carcinoma & $1.26 \pm 0.33$ & $1.26 \pm 0.74$ & $1.18 \pm 0.23$ & * & * \\
\hline $\begin{array}{l}\text { Thymic } \\
\text { hyperplasia }\end{array}$ & * & * & * & $1.86 \pm 0.17$ & $\begin{array}{l}35.51 \pm \\
12.51\end{array}$ \\
\hline Lymphoma & $1.11 \pm 0.21$ & $2.35 \pm 0.05$ & * & * & * \\
\hline
\end{tabular}

Apparent diffusion coefficient (ADC) and SII (which is obtained from the dual-echo technique without the need of an internal control and reflects the lipid content of the tissue) are important quantitative parameter of MRI [28, 29]. Table 5 showed quantitative assessment of MRI in different thymic masses. Quantitative evaluation of MRI and CT in different types of thymoma was showed in Table 6 . Of note, Priola et al. reported SII of thymic hyperplasia was $0.38 \pm 24.73 \%$ [25].

Table 6. Quantitative assessment of $M R I$ and $C T$ in different types of thymoma.

\begin{tabular}{llll}
\hline & Priola et al.2015 & Priola et al. 2016 & Priola et al. 2016 \\
\hline Thymoma & ADC $^{*}\left(\times 10-3 \mathrm{~mm}^{2} / \mathrm{s}\right)$ & $\mathrm{SII} * *(\%)$ & Radiodensity $(\mathrm{HU})^{* * *}$ \\
Early thymoma & $1.43 \pm 0.26$ & $-0.95 \pm 3.65$ & $33.64 \pm 10.82$ \\
Advanced thymoma & $1.31 \pm 0.31$ & $0.91 \pm 4.4$ & $32.31 \pm 11.43$ \\
\hline$*$ :Apparent Diffusion Coefficient & \\
$* * *$ SII is obtained from the dual-echo technique without the need of an internal \\
control and reflects the lipid content of the tissue. \\
$* * * *$ Radiodensity in Hounsfield unit
\end{tabular}

\section{Comparison of diagnostic accuracy between CT and MRI}

Diagnostic performance of CT and MRI in ROC curve was shown in Figure 1. At quantitative measurement, the sensitivity of CT and MRI were both $100 \%$, while the specificities were $75 \%$ and $80 \%$, respectively. Additionally, area under curve (AUC) of CT is 0.875 [95\%CI: $0.473,0.997]$ and that of MRI is 0.880 [95\% CI: 0.531, 0.995].

\section{Discussion}

Thymoma is the most common type of neoplasms in the mediastinum and those with low risk and in early stage usually have good prognosis [9, 30]. Thymectomy is the mainstay of therapy of thymic masses and radiotherapy and chemotherapy are often as (neo)adjuvant and palliative procedures[8, 9]. Meanwhile, if thymoma or thymic carcinoma was discovered earlier, the rate of whole resection is high. Therefore, it is crucially important for thoracic surgeons to obtain accurate pre-operative identifications of staging and histological types of thymic masses.

Indeed, thymic lesions are uncommon and relative studies are rare compared with diseases involving other organs like lung. Therefore, only eight studies were finally included in our study in recent five years. Intriguingly, we found that the lower degree in which thymic masses invaded, and the higher CT attenuation were demonstrated. The performance of diagnosis of MRI was a little superior than that of CT in thymomas, thymic cysts and thymic hyperplasia, whereas differentiating thymic carcinomas, lymphomas/germ cell tumors and other masses were inferior to that of CT. In our study, ROC analysis was used to evaluate the diagnostic capability of CT and MRI, and they were both powerful modalities for thymic lesions. Of note, we 
found that CT and MRI both have high sensitivity. AUC of CT and MRI was 0.875 and 0.880, respectively. Thus, we can safely conclude that the diagnostic capability of MRI is superior to that of CT in differentiating certain types of thymic masses combining with the results of statistical correctness of included studies.
CT is generally considered to be superior to MRI in the evaluation and characterization of most anterior mediastinal masses [15]. However, the versatility of MRI is superior to that of CT in certain scenarios, especially in the differentiation of thymic cysts [2]. Indeed, MRI is a promising modality to differentiate thymic masses because of high contrast resolution, no

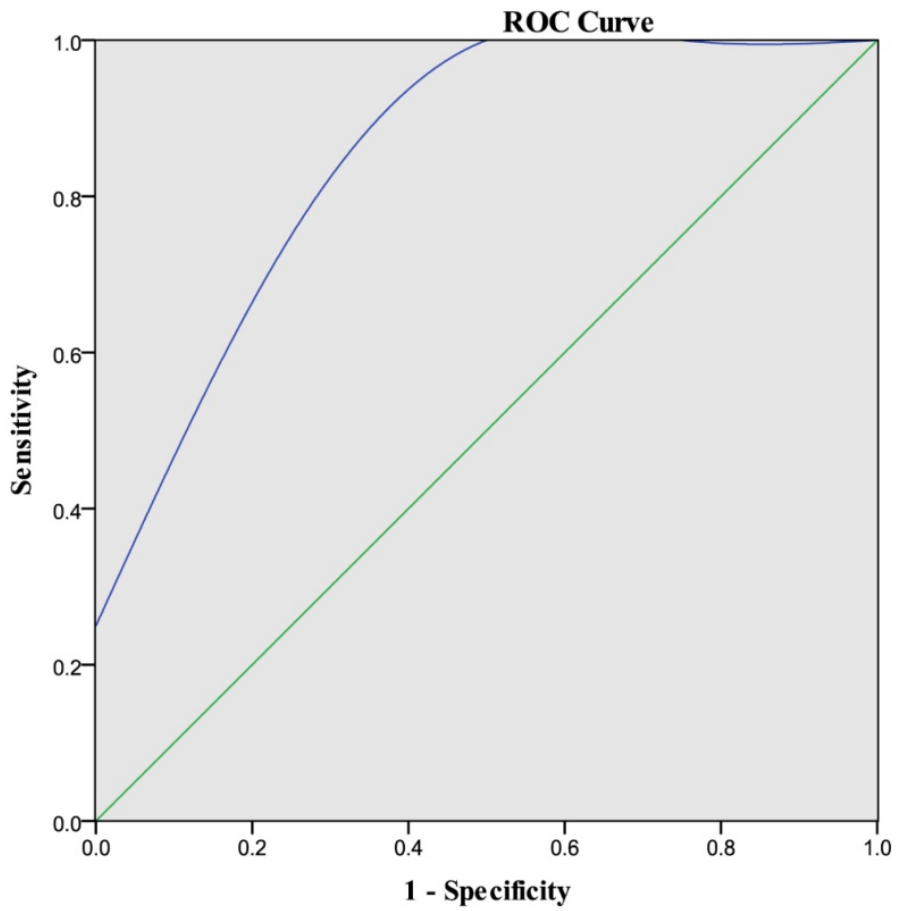

$\underbrace{\mathrm{CT}}_{\text {Reference Line }}$

AUC:0.875

Sensitivity: $100 \%$

Specificity: $75 \%$

SD:0.144

$\mathrm{P}=0.0094$

B

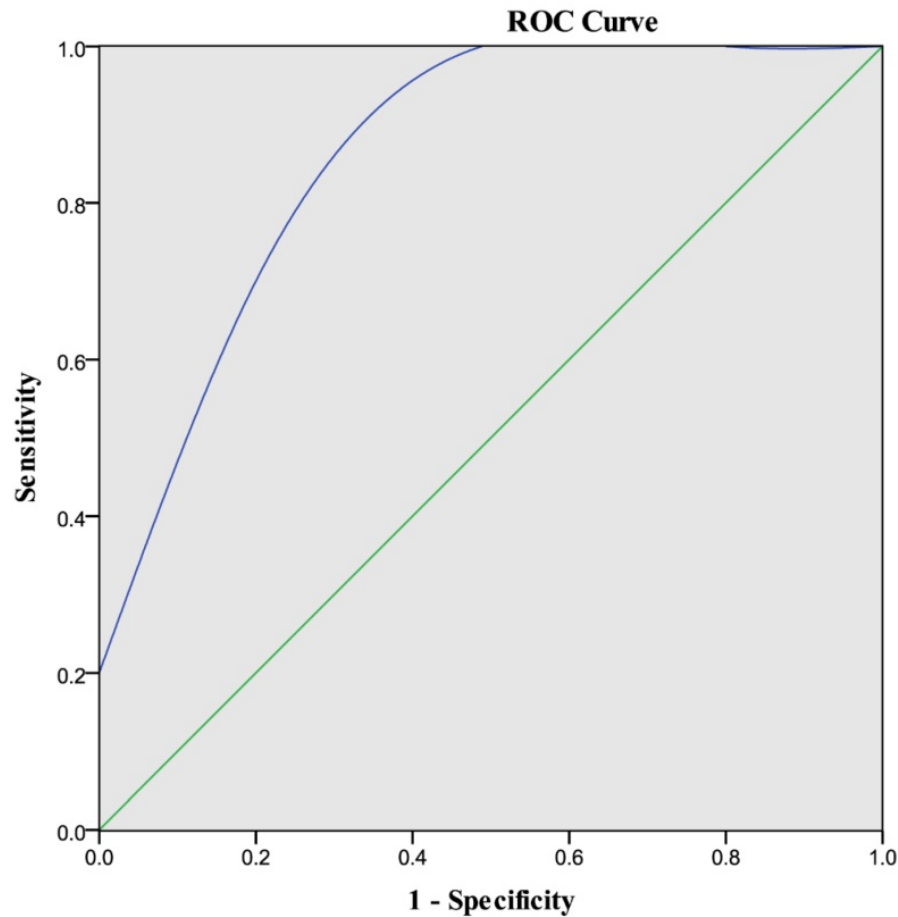

$\sim \mathrm{MRI}$

Reference Line

AUC:0.880

Sensitivity: $100 \%$

Specificity: $80 \%$

SD:0.130

$\mathrm{P}=0.0034$

Figure 1. Diagnostic performance of CT and MRI in ROC curve. (The sensitivity of CT and MRI were both $100 \%$, while the specificity were $75 \%$ and $80 \%$, respectively. AUC (area under curve) of CT is 0.875 [ $95 \% \mathrm{Cl}: 0.473,0.997]$ and that of $\mathrm{MRI}$ is 0.880 [95\% Cl: $0.531,0.995]$.) 
radiation exposure [28]. Chemical shift MRI (CS-MRI) is highly accurate in distinguishing thymomas from thymic lymphoid hyperplasia or normal thymus. However, it is not useful in differentiating between thymic lymphoid hyperplasia and normal thymus [28, 29]. Diffusion-weighted magnetic resonance imaging (DWI) could not only distinguish benign mediastinal tumors from malignant ones, but also distinguish among low-risk thymoma, high-risk thymoma and other malignant mediastinal tumors [16, 24]. DWI did not show excellent predictive ability of distinguishing early diseases from advanced ones based on Masaoka-Koga system [16, 31].

Priola et al. demonstrated that CS-MRI was more reliable than CT for distinguishing thymomas from thymic lymphoid hyperplasia and normal thymus through both qualitative and quantitative analyses, especially in evaluating patients with myasthenia gravis [25]. In qualitative assessment, MRI had higher accuracy than CT, and signal intensity of MRI was significantly different between groups [25]. Seki et al. [27] and Tomiyama et al. [2] both found that DWI is equal to $\mathrm{CT}$ in the diagnosis of thymomas. Additionally, Benveniste et al. concluded that the performance of MRI is similar to that of CT in distinguishing patients with advanced stage TETs on the 6th International Thymic Malignancy Interest Group Annual Meeting in October 2015 (www.itmig2015.org).

Our study demonstrated that the diagnostic utility of CT was inferior to that of MRI in detecting thymomas, thymic cysts or thymic hyperplasia, which was consistent with outcomes of some researchers [16, $24,28,29]$. CT possesses advantages in the spatial, temporal resolution convenience and time-short as the first choice of detection of thymic masses, while MRI does well in detecting the relation between masses and theirs surrounding tissues especially connective tissues invasion such as vessels. In addition, we still cannot ignore that confounding diagnosis may occur when different masses performed same images. For instance, when rebound thymic hyperplasia occurs in patients who previously underwent chemotherapy for malignancy, distinguishing thymic rebound from recurrent neoplasm may be difficult [18]. Thymoma and thymic carcinomas may exhibit cystic changes both on CT and MRI [2]. Moreover, different diseases can co-exist such as hyperplasia and cysts, and sometimes this mixed lesion looks simply like a malignant tumor. Practically, differentiating thymomas from lymphomas or thymic hyperplasia was often difficult $[26,32]$. Some researchers even demonstrated that CT could predict subtypes of TETs [33], but more evidence is required.
To assess whether pre-operative CT could identify those patients who should or not receive surgical therapies, Hayes et al. designed a study to evaluate the ability of CT in predicting the resectability of thymomas according to imaging characteristics. There were several preoperative imaging features including lobulated tumor contour and tumor sizes which showed significant association with an incomplete surgical resection or late stage thymomas on univariate analysis [34]. On multivariate analysis, greater than or equal to $50 \%$ abutment of the circumference of an adjacent vessel on CT was statistically associated with an incomplete resection or late stage thymomas [34].

Still, our study has some limitations. For example, the number of included studies was small due to the rarity of thymic masses. Next, further differentiation between contrast $\mathrm{CT}$ and non-contrast CT cannot be realized because of lack of relevant studies. Also, comparisons in different functional modes of MRI are unavailable. Therefore, more researches are warranted to further elucidate the capabilities of MRI to predict whether thymic lesions need to be resected.

\section{Conclusion and future expectation}

The diagnostic accuracy of MRI is superior to CT in detecting thymomas, thymic cysts or thymic hyperplasia and that of CT and MRI is still unclear in differentiating thymic carcinomas and lymphomas/ germ cell tumors and mere image analysis is insufficient for accurate diagnosis of thymic masses. Imaging plays a crucial role in diagnosis, staging and even treatment of patients with thymic masses, but both CT and MRI have their own shortcomings and cannot provide accurate pre-operative diagnosis. Therefore, it may be the future direction of diagnosing thymic diseases that trying to find more reliable biomarkers.

\section{Abbreviations}

TETs, thymic epithelial tumors; AUC, area under curve; ADC, apparent diffusion coefficient; MRI, magnetic resonance imaging; $\mathrm{CT}$, computed tomography.

\section{Acknowledgements}

This study was funded by Talent Funding Program of Zhongshan Hospital of Fudan University. In addition, we were grateful for the help from Zehuan Li (Department of General Surgery, Zhongshan Hospital, Fudan University) and Peng Wu (Key Laboratory of Health Technology Assessment of Ministry of Health, Collaborative Innovation Center 
of Social Risks Governance in Health, School of Public Health, Fudan University).

\section{Competing Interests}

The authors have declared that no competing interest exists.

\section{References}

1. Strollo DC, Rosado de Christenson ML, Jett JR. Primary mediastinal tumors. Part 1: tumors of the anterior mediastinum. Chest. 1997; 112: 511-22.

2. Tomiyama N, Honda O, Tsubamoto M, Inoue A, Sumikawa H, Kuriyama K, et al. Anterior mediastinal tumors: diagnostic accuracy of CT and MRI. European journal of radiology. 2009; 69: 280-8.

3. Goldstein AJ, Oliva I, Honarpisheh $\mathrm{H}$, Rubinowitz A. A tour of the thymus: a review of thymic lesions with radiologic and pathologic correlation. Canadian Association of Radiologists journal = Journal l'Association canadienne des radiologistes. 2015; 66: 5-15

4. Venuta F, Anile M, Diso D, Vitolo D, Rendina EA, De Giacomo T, et al Thymoma and thymic carcinoma. European journal of cardio-thoracic surgery : official journal of the European Association for Cardio-thoracic Surgery. 2010; 37: 13-25

5. Ettinger DS, Riely GJ, Akerley W, Borghaei H, Chang AC, Cheney RT, et al. Thymomas and thymic carcinomas: Clinical Practice Guidelines in Oncology. Journal of the National Comprehensive Cancer Network: JNCCN. 2013; 11: $562-76$

6. Benveniste MF, Rosado-de-Christenson ML, Sabloff BS, Moran CA, Swisher SG, Marom EM. Role of imaging in the diagnosis, staging, and treatment of thymoma. Radiographics : a review publication of the Radiological Society of North America, Inc. 2011; 31: 1847-61; discussion 61-3.

7. Filosso PL, Yao X, Ruffini E, Ahmad U, Antonicelli A, Huang J, et al. Comparison of outcomes between neuroendocrine thymic tumours and other subtypes of thymic carcinomas: a joint analysis of the European Society of Thoracic Surgeons and the International Thymic Malignancy Interest Group. European journal of cardio-thoracic surgery : official journal of the European Association for Cardio-thoracic Surgery. 2016; 50: 766-71.

8. Kondo K. Optimal therapy for thymoma. The journal of medical investigation : JMI. 2008; 55: 17-28.

9. Kondo K. Therapy for thymic epithelial tumors. General thoracic and cardiovascular surgery. 2014; 62: 468-74

10. Romi F. Thymoma in myasthenia gravis: from diagnosis to treatment. Autoimmune diseases. 2011; 2011: 474512 .

11. Zhao Y, Chen H, Shi J, Fan L, Hu D, Zhao H. The correlation of morphological features of chest computed tomographic scans with clinical characteristics of thymoma. European journal of cardio-thoracic surgery : official journal of the European Association for Cardio-thoracic Surgery. 2015; 48: 698-704.

12. Ried M, Marx A, Gotz A, Hamer O, Schalke B, Hofmann HS. State of the art: diagnostic tools and innovative therapies for treatment of advanced thymoma and thymic carcinoma. European journal of cardio-thoracic surgery : official journal of the European Association for Cardio-thoracic Surgery. 2016; 49: 1545-52

13. Andreini D, Pontone G, Dainese L, Formenti A, Mushtaq S, Cappai A, et al. Preoperative assessment of thymoma: evaluation of mediastinal arterial anatomy by cardiac multidetector computed tomography. Journal of thoracic imaging. 2009; 24: 31-3.

14. Marom EM. Advances in thymoma imaging. Journal of thoracic imaging. 2013; 28: 69-80; quiz 1-3.

15. Carter BW, Benveniste MF, Truong MT, Marom EM. State of the Art: MR Imaging of Thymoma. Magnetic resonance imaging clinics of North America. 2015; $23: 165-77$.

16. Abdel Razek AA, Khairy M, Nada N. Diffusion-weighted MR imaging in thymic epithelial tumors: correlation with World Health Organization classification and clinical staging. Radiology. 2014; 273: 268-75.

17. Priola AM, Priola SM. Imaging of thymus in myasthenia gravis: from thymic hyperplasia to thymic tumor. Clinical radiology. 2014; 69: e230-45.

18. Takahashi K, Al-Janabi NJ. Computed tomography and magnetic resonance imaging of mediastinal tumors. Journal of magnetic resonance imaging : JMRI. 2010; 32: 1325-39.

19. Kondo K, Yoshizawa K, Tsuyuguchi M, Kimura S, Sumitomo M, Morita J, et al. WHO histologic classification is a prognostic indicator in thymoma. The Annals of thoracic surgery. 2004; 77: 1183-8.

20. Koga K, Matsuno Y, Noguchi M, Mukai K, Asamura H, Goya T, et al. A review of 79 thymomas: modification of staging system and reappraisal of conventional division into invasive and non-invasive thymoma. Pathology international. 1994; 44: 359-67.

21. Yabuuchi H, Matsuo Y, Abe K, Baba S, Sunami S, Kamitani T, et al. Anterior mediastinal solid tumours in adults. characterisation using dynamic contrast-enhanced MRI, diffusion-weighted MRI, and FDG-PET/CT. Clinical radiology. 2015; 70: 1289-98.

22. Chang S, Hur J, Im DJ, Suh YJ, Hong YJ, Lee HJ, et al. Volume-based quantification using dual-energy computed tomography in the differentiation of thymic epithelial tumours: an initial experience. European radiology. 2017; 27: 1992-2001.

23. Araki T, Sholl LM, Gerbaudo VH, Hatabu H, Nishino M. Intrathymic cyst: clinical and radiological features in surgically resected cases. Clinical radiology. 2014; 69: 732-8

24. Usuda K, Maeda S, Motono N, Ueno M, Tanaka M, Machida Y, et al. Diffusion Weighted Imaging Can Distinguish Benign from Malignant Mediastinal Tumors and Mass Lesions: Comparison with Positron Emission Tomography. Asian Pacific journal of cancer prevention : APJCP. 2015; 16: 6469-75.

25. Priola AM, Priola SM, Gned D, Giraudo MT, Fornari A, Veltri A. Comparison of CT and chemical-shift MRI for differentiating thymoma from non-thymomatous conditions in myasthenia gravis: value of qualitative and quantitative assessment. Clinical radiology. 2016; 71: e157-69.

26. Ackman JB, Verzosa S, Kovach AE, Louissaint A, Jr., Lanuti M, Wright CD, et al. High rate of unnecessary thymectomy and its cause. Can computed tomography distinguish thymoma, lymphoma, thymic hyperplasia, and thymic cysts? European journal of radiology. 2015; 84: 524-33.

27. Seki S, Koyama H, Ohno $Y$, Nishio M, Takenaka D, Maniwa $Y$, et al. Diffusion-weighted MR imaging vs. multi-detector row CT: Direct comparison of capability for assessment of management needs for anterior mediastinal solitary tumors. European journal of radiology. 2014; 83: 835-42.

28. Priola AM, Gned D, Veltri A, Priola SM. Chemical shift and diffusion-weighted magnetic resonance imaging of the anterior mediastinum in oncology: Current clinical applications in qualitative and quantitative assessment. Critical reviews in oncology/hematology. 2016; 98: 335-57.

29. Priola AM, Priola SM, Giraudo MT, Gned D, Giardino R, Marci V, et al. Chemical-shift and diffusion-weighted magnetic resonance imaging of thymus in myasthenia gravis: usefulness of quantitative assessment. Investigative radiology. 2015; 50: 228-38.

30. Casey EM, Kiel PJ, Loehrer PJ, Sr. Clinical management of thymoma patients. Hematology/oncology clinics of North America. 2008; 22: 457-73.

31. Priola AM, Priola SM, Giraudo MT, Gned D, Fornari A, Ferrero B, et al. Diffusion-weighted magnetic resonance imaging of thymoma: ability of the Apparent Diffusion Coefficient in predicting the World Health Organization (WHO) classification and the Masaoka-Koga staging system and its prognostic significance on disease-free survival. European radiology. 2016; 26: 2126-38.

32. Marom EM. Imaging thymoma. Journal of thoracic oncology : official publication of the International Association for the Study of Lung Cancer. 2010; 5: S296-303.

33. Hu YC, Wu L, Yan LF, Wang W, Wang SM, Chen BY, et al. Predicting subtypes of thymic epithelial tumors using CT: new perspective based on a comprehensive analysis of 216 patients. Scientific reports. 2014; 4: 6984

34. Hayes SA, Huang J, Plodkowski AJ, Katzen J, Zheng J, Moskowitz CS, et al. Preoperative computed tomography findings predict surgical resectability of thymoma. Journal of thoracic oncology : official publication of the International Association for the Study of Lung Cancer. 2014; 9: 1023-30. 